\title{
Influence of Temperature and Noise on Subthreshold Signal Propagation in Feedforward Neural Network
}

\section{Ming Yi ( $\sim$ mingyi@cug.edu.cn )}

China University of Geosciences School of Mathematics and Physics

\section{Shiqi Dai}

China University of Geosciences School of Mathematics and Physics

Lulu Lu

China University of Geosciences School of Mathematics and Physics

\section{Zhouchao Wei}

China University of Geosciences School of Mathematics and Physics

\section{Yuan Zhu}

China University of Geosciences School of Automation

\section{Research Article}

Keywords: Signal propagation , Temperature, Noise , Feedforward neural network

Posted Date: October 18th, 2021

DOl: https://doi.org/10.21203/rs.3.rs-978086/v1

License: (9) This work is licensed under a Creative Commons Attribution 4.0 International License. Read Full License 


\title{
Influence of temperature and noise on subthreshold signal propagation in feedforward neural network
}

Shiqi Dai $・$ Lulu Lu • Zhouchao Wei $・$ Yuan Zhu • Ming Yi

S. Dai · L. Lu · Z. Wei · M. Yi (*)

School of Mathematics and Physics,

China University of Geosciences, Wuhan 430074, Hubei, China,

e-mail:mingyi@cug.edu.cn

Y. Zhu

Engineering Research Center of Intelligent Technology for Geo-Exploration, Ministry of Education,

Hubei Key Laboratory of Advanced Control and Intelligent Automation for Complex Systems,

School of Automation, China University of Geosciences, Wuhan 430074, Hubei, China,

Key Laboratory of Computational Neuroscience and Brain-Inspired Intelligence, Ministry of Education,

Fudan University, Shanghai 200433, China

\begin{abstract}
Temperature is an important environmental factor that all creatures depend on. Under the appropriate temperature, the neural system shows good biological performance. Based on an improved Hodgkin-Huxley (HH) neuron model considering temperature and noise, the ten-layers pure excitatory feedforward neural network and the ten-layers excitatory-inhibitory (EI) neural network are constructed to study the subthreshold signal propagation. It's found that increasing temperature can restrain the signal propagation, and raise the noises intensity threshold where the failed signal propagation can transform into succeed signal propagation. Under the large noise, the signal propagation in network in different temperatures exhibits different anti-noise capabilities. There exists the saturation value of interlayer connection probability, that is, the signal propagation maintains constant when interlayer connection probability beyond a certain value. Moreover, in EI network with large noise, the network's intrinsic oscillation activity will completely cover subthreshold signal, and block the signal propagation. The jumping phenomenon in the value of fidelity, which measures the similarity between input signal and output signal, appears in both pure excitatory network and EI network. This paper provides potential value for understanding the regulation of both temperature and noise in information propagation in neural network.
\end{abstract}

Keywords Signal propagation $\cdot$ Temperature $\cdot$ Noise $\cdot$ Feedforward neural network

\section{Introduction}


Signal transmission and processing in the neural system are affected by many factors, such as temperature, noise, electromagnetic induction, connection structure, external input signal [1-12]. Temperature is an inevitable influencing factor, which regulates the information coding and processing by affecting the opening and closing probabilities of ion channels on neuron membranes [1]. Experimental and theoretical studies about the temperature's effects on neuron dynamics has been carried out recently. The temperature dependence of action potential parameters [2] and conduction of action potential [3] were studied in vitro experiments. A small fluctuation in temperature may cause diseases in the neural system. Extreme temperatures can affect the physiological function of neurons through affecting neuronal cell, leading to Parkinson's disease [5]. The febrile seizures occurs commonly in young children under the pathologically high temperature [6]. Hypo-metabolism under low temperature may be the reason that triggers cognitive decline and induces neurodegeneration [7]. Lu et al [8] studied the effects of both temperature and electromagnetic induction on the neurons' electrical activities, and found an Arnold's tongue area on the plane of external current and temperature parameters. Xing et al [9] analyzed the bifurcation of the Morris-Lecar neuron model for two kinds of representative neurons with temperature-sensitive calcium and leak current, and found the switching between different firing patterns of neurons. Ding et al [10] studied the influence of temperature on the propagation of action potential on myelin axon, and found that the lower temperature is beneficial for action potential's propagate in myelin axon. Xu et al. [11] found that changing temperature with thermistors can control the neuron dynamics.

In addition to temperature, noise is also a non-negligible factor affecting the neural system. Noise is ubiquitous in the neural system, caused by thermal effects, activity of ion channels and etc.[13,14]. The appropriate noise plays an important role in information transmission and detection, which can induce stochastic resonance (SR), and optimizing the detection and propagation of weak signals [15-18]. For example, there is an optimal Gaussian colored noise intensity under which the subthreshold signal can propagate stably in feedforward neural network (FFN) [19]. Similarly, the optimal Gaussian white noise intensity was also found for signal propagation in FFN composed of hybrid neurons [20]. Guo et al. found that there exists an appropriate noise intensity which can enhance the auditory effect through establishing nonlinear resonance, and benefit for the signal detection in auditory system [21].

Subthreshold signal is generated by weak activities in the environment, and it is too easily hidden in noise to be detected [22]. It is widely believed that the neurons encode and transmit information by firing rate, firing time precision and etc. [23-25], and information is thought to be stored in the peak interval of neuron spikes sequence (ISIs) [26]. Because the brain has a highly modular structure, information is processed and transmitted between different modules, so information carried by the precise timing of spikes would have to propagate through a hierarchy cortical area [27]. 
This modular structure can be simulated by multi-layer feedforward neural network, which is divided into the input layer, the middle layer and the output layer [28]. Neural networks have many applications in the field of artificial intelligence, which makes the neural network has received a lot of attention from researchers in different disciplines in recent years. From an experiment view, the hippocampal neurons of rat were applied to investigate the signal propagation in feedforward neural network $[29,30]$. From a mathematical point of view, the stability of neural networks under impulsive disturbance and the condition of exponential synchronization of neural networks under feedback control were studied by using mathematical tools such as Lyapunov function method and linear matrix inequalities (LMIs) [31,32].

In addition, the impact of the feedforward neural network's architecture structure on signal transmission has been noticed widely. For example, increasing the interlayer connection probability can optimize the signal transmission, but the study have shown that too large interlayer connection probability is actually a waste [33]. Considering the interlayer connection way, the research found that when the interlayer connection probability of feedforward network follows exponential distribution, the synchronous spike chain propagates best [34]. Previous researchers mainly studied the signal propagation in pure excitatory feedforward neural network, actually, there exist excitatory and inhibitory neurons in neural system in approximate ratio of 4:1 [35]. The different connections between two types of neurons, e.g., recurrent connections and feedback connections, form the complex network structure, that have been confirmed in many biological experiments [36-39]. Different from pure excitatory feedforward network, the intralayer connections between excitatory and inhibitory neurons can lead to varied intrinsic activities (or spontaneous activities) of EI network, then intrinsic activities effect the signal transmission. In addition, the ratio of inhibitory to excitatory synaptic strength has been used to determine which type of neuron is dominant. When inhibitory neurons are dominant, the intrinsic activity of the network is asynchronous irregular (AI), otherwise, the intrinsic firing activity of the network is synchronous regular (SR) [40]. That is, there exist the delicate balance between excitatory and inhibitory neurons to maintain the normal function of the neural system, and the balance is regulated by connection and synaptic parameters $[41,42]$. In essence, when the inhibitory and excitatory inputs cancel out, the membrane potential of neurons fluctuates randomly, resulting in irregular discharges. When neurons share EI inputs that do not cancel each other out, the network tends to synchronize [43]. The spontaneous activity of the network can be utilized rationally, when the frequency of the input signal matches the intrinsic firing oscillation frequency of the network, the signal can be amplified and effectively transmitted to the output layer, this is the resonance phenomenon [43-46]. It has been found that the EI balance can be regulated by temperature. Increasing temperature has opposite effects on changing the firing rates of 
excitatory and inhibitory neurons, consequently, causing EI imbalance and lead to the diseases [47].

In this paper, based on the modified Hodgkin-Huxley model considering temperature and noise, the pure excitatory and EI feedforward ten-layer neural network are constructed. We calculate power norm (or fidelity $C_{0}$ ) and time lag $\left(\tau_{d}\right)$ to quantify the signal propagation. On the one part, for the pure excitatory feedforward ten-layer neural network, the effects of temperature, noise intensity, and interlayer connection probability on the signal propagation are investigated. On the other part, for the EI feedforward ten-layer neural network, the influence of network structure, temperature and noise intensity on subthreshold signal transmission are studied. Regarding the structure of the paper, the models for two type feedforward neural networks and the synaptic model are given in Sect. 2. The simulation results and analysis are shown in Sect. 3. In Sect.4, we have made a summary.

\section{Models and methods}

\subsection{Network Model}

As shown in Fig.1, we constructed a 10-layer feedforward neural network with pure excitatory neurons, each layer contains 1000 excitatory neurons, and the $j$-th neuron in layer $i$ is represented by subscript $(i, j)$. The first layer is the input layer, where each neuron receives the same excitatory subthreshold signal and connects with the next layer. First of all, we fixed the interlayer connection probability $\left(P_{\text {inter }}\right)$ as 0.2 , that is, except for the input layer, each neuron in each layer receives excitatory input from 200 randomly selected neurons in the previous layer, and there is no intralayer connection in each layer. Then, $P_{\text {inter }}$ will be varied gradually to research the effects of interlayer connection probability on signal propagation. In addition, all neurons are affected by noise.

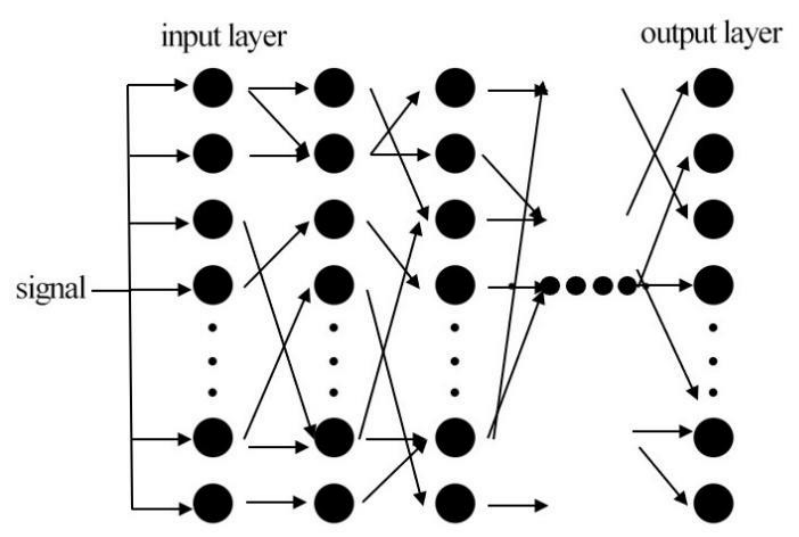

Fig.1 Pure excitatory 10-layer feedforward neural network, each black circle represents an excitatory neuron. The neurons in input layer receive the subthreshold signal, the signal is propagated and amplified through the middle layer 
to the output layer

As shown in Fig.2, the EI feedforward neural network with ten layers of tight intralayer connections and sparse interlayer connections is constructed. Each layer has 800 excitatory neurons and 200 inhibitory neurons, where the excitatory or inhibitory neurons are distinguished by the parameters of their synaptic model. There are excitatoryexcitatory (E-E) connections, excitatory-inhibitory (E-I) connections, inhibitory-excitatory (I-E) connections, and inhibitory-inhibitory (I-I) connections in each layer, and their connection probabilities are: $P_{E E}=0.25, P_{E I}=P_{I E}=P_{I I}=$ 0.3. The excitatory neurons of each layer connect to the excitatory and inhibitory neurons of the next layer, and the interlayer connection probability is $P_{\text {inter }}=0.05$. Similarly, the first layer is the input layer, where each neuron receives the subthreshold signal, and the tenth layer is the output layer. EI neural network is more complex and more real than pure excitatory network, the excitatory synapses in EI network can stimulate the firing of neurons, while the inhibitory synapses can inhibit the firing of neurons. All neurons are affected by noise.

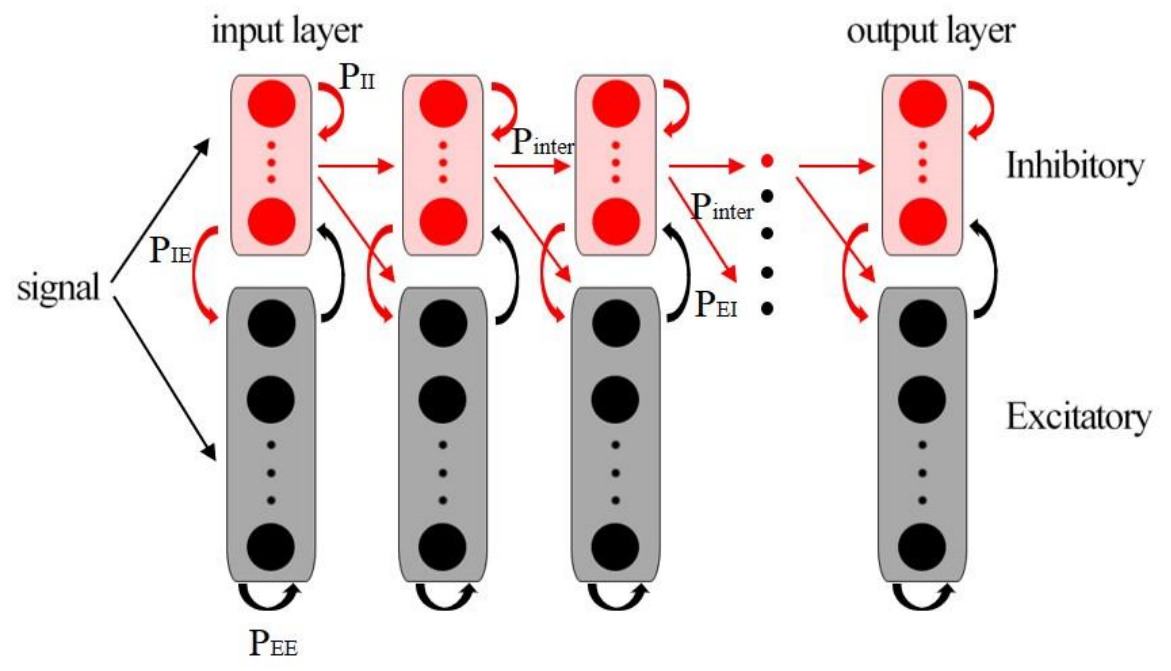

Fig.2 10-layers excitatory-inhibitory feedforward neural network, the red circles represent inhibitory neurons, and the black circles represent excitatory neurons. There are E-E connection, E-I connection, I-E connection and I-I connection inside each layer. The excitatory neurons connected to the both inhibitory and excitatory neurons in the next layer

\subsection{Neuron Model}

Hodgkin-Huxley $(\mathrm{HH})$ model is a realistic model that can realistically reflect various firing patterns of neurons [48]. We use the modified $\mathrm{HH}$ model to simulate the neurons affected by temperature. The specific expression is as follows: 


$$
\begin{gathered}
\frac{d V_{i j}}{d t}=-\frac{1}{C_{m}}\left[g_{K} n_{i j}^{4}\left(V_{i j}-V_{K}\right)+g_{N a} m_{i j}^{3} h_{i j}\left(V_{i j}-V_{N a}\right)+g_{L}\left(V_{i j}-V_{L}\right)\right]+I_{i j}^{s y n}(t)+I_{0}+S(t)+\xi_{i j}(t), \\
\frac{d x_{i j}}{d t}=\alpha_{x}\left(1-x_{i j}\right)-\beta_{x} x_{i j},\left(x_{i j}=m_{i j}, n_{i j}, h_{i j}\right),
\end{gathered}
$$

Where the subscript $(i, j)$ represents the $j$-th cell in the $i$ layer, $V_{i j}$ represents the membrane potential of the $(i, j)$ neuron, and $C_{m}$ represents the membrane capacitance. $g_{N a}, g_{K}, g_{L}$ represent the maximum conductance per surface unit of sodium, potassium and leakage current, respectively. $V_{N a}, V_{K}, V_{L}$ are the corresponding reversal potential. $n$ is the activation probability of potassium ion channel, $m$ and $h$ are the inactivation probability and activation probability of sodium ion channel respectively. $I_{i j}^{\text {yn }}(\mathrm{t})$ is the synaptic current received by the $(i, j)$ neuron, $I_{0}$ is the external constant current, $I_{0}=1 \mathrm{~mA} . \xi_{i j}(\mathrm{t})$ is Gaussian white noise, which satisfies $\left\langle\xi_{i j}(t)\right\rangle=0,\left\langle\xi_{i j}(t) \xi_{i j}\left(t^{\prime}\right)\right\rangle=2 D \delta\left(t-t^{\prime}\right)$, where $D$ represents the intensity of the noise.

$S(\mathrm{t})$ represents subthreshold signal, which is expressed as the excitatory post-synaptic current (EPSC). EPSC is produced by the excitatory neurotransmitter released by the pre-synapse acting on the membrane of the post-synapse. Only neurons in the first layer receive the subthreshold signal, and its expression is as follows [49]:

$$
\begin{aligned}
& S(t)=g_{\text {in }}\left(E_{s y n}-V_{i j}\right), \\
& g_{\text {in }}=A \frac{t}{\tau} \exp \left(1-\frac{t}{\tau}\right),
\end{aligned}
$$

The signal is injected when $t \geq 0$. The parameter $A=0.022 \mu \mathrm{V} / \mathrm{cm}^{2}$, indicating that the signal is the subthreshold signal. The reversal potential is $E_{s y n}=0 \mathrm{mV}$, and the time constant is $\tau=2.0 \mathrm{~ms}$. The variable $\alpha_{x}, \beta_{x}$ in Eq. (2) are rate function of the gated variable $x_{i j}$, and their expressions are as follows:

$$
\begin{gathered}
\alpha_{n_{i j}}=\frac{0.01 \phi(T)\left(V_{i j}+55\right)}{1-\exp \left[-\left(V_{i j}+55\right) / 10\right]}, \\
\beta_{n_{i j}}=0.125 \phi(T) \exp \left[-\left(V_{i j}+65\right) / 80\right], \\
\alpha_{m_{i j}}=\frac{0.1 \phi(T)\left(V_{i j}+40\right)}{1-\exp \left[-\left(V_{i j}+40\right) / 10\right]}, \\
\beta_{m_{i j}}=4 \phi(T) \exp \left[-\left(V_{i j}+65\right) / 18\right], \\
\alpha_{h_{i j}}=0.07 \phi(T) \exp \left[-\left(V_{i j}+65\right) / 20\right],
\end{gathered}
$$




$$
\beta_{h_{i j}}=\frac{\phi(T)}{1+\exp \left[-\left(V_{i j}+35\right) / 10\right]},
$$

With

$$
\phi(T)=3^{\left(T-6.3^{\circ} \mathrm{C}\right) / 10^{\circ} \mathrm{C}}
$$

Here, $T$ represents temperature in degrees Celsius, the temperature function refers to the reference [8]. The values of other parameters are: $V_{N a}=50.0 \mathrm{mV}, V_{K}=-77.0 \mathrm{mV}, V_{L}=-54.4 \mathrm{mV}, g_{K}=36.0 \mathrm{mS} / \mathrm{cm}^{2}, g_{N a}=120.0 \mathrm{mS} / \mathrm{cm}^{2}, g_{L}=$ $0.3 \mathrm{mS} / \mathrm{cm}^{2}, C_{m}=1.0 \mu \mathrm{F} / \mathrm{cm}^{2}[22]$.

\subsection{Synaptic Model}

We use $\alpha$ function to simulate the post-synaptic current response. The $\alpha$ function, originally proposed by Rall, provides a sequence diagram of the current in the post-synaptic membrane over time after being stimulated by the firing neuron $[50,51]$. The synaptic current received by the neuron $(i, j)$ is:

$$
I_{i j}^{\text {inter }}=-\frac{1}{N_{i j}^{\text {inter }}} \sum_{p=1}^{N_{i j}^{\text {inter }}} g_{\text {inter }} \alpha\left(t-t_{i-1, p}\right)\left(V_{i j}-V_{s y n}\right)
$$

And,

$$
\begin{gathered}
I_{i j}^{\text {intra }}=-\frac{1}{N_{i j}^{\text {intra }}} \sum_{p=1}^{N_{i \text { inta }}^{\text {int }}} g_{\text {intra }} \alpha\left(t-t_{i, p}\right)\left(V_{i j}-V_{s y n}\right), \\
I_{i j}^{s y n}=I_{i j}^{\text {inter }}+I_{i j}^{\text {intra }}, \\
\alpha(t)=\frac{t}{\tau} \exp \left(-\frac{t}{\tau}\right) \Theta(t),
\end{gathered}
$$

Where, eq. (11) calculates the interlayer connection current, the number $N_{i j}^{\text {inter }}$ represents the total number of neurons in the previous layer connected to the neuron $(i, j), t_{i-1, p}$ represents the firing moment of the $p$-th neuron connected to neuron $(i, j)$ in the $i$-1layer. The term $g_{\text {inter }}$ is synaptic wight, representing the strength of the interlayer connection. There is only excitatory connection between layers, so the reversal potential $V_{s y n}=0 \mathrm{mV}$ in eq. (11). Eq.

$$
I_{i j}^{\text {intra }}=-\frac{1}{N_{i j}^{\text {intra }}} \sum_{p=1}^{N_{i j}^{\text {inta }}} g_{\text {intra }} \alpha\left(t-t_{i, p}\right)\left(V_{i j}-V_{s y n}\right),
$$

(12) represents the intralayer connection current composed of excitatory current and inhibitory current. Similarly, $N_{i j}^{\text {inta }}$ is the total number of neurons connected to neuron $(i, j), t_{i, p}$ represents the firing moment of the $p$-th neuron connected to $(i, j)$ in the $i$ layer. The other parameters are: $g_{\text {intra }}=g_{\text {intra }}^{\text {exc }}$ $=0.6, V_{s y n}=V_{s y n}^{x x c}=0 \mathrm{mV}$ for excitatory synapses, $g_{\text {intra }}=g_{i n t r a}^{\text {inh }}=0.6, V_{s y n}=V_{s y n}^{\text {inh }}=-80 \mathrm{mV}$ for inhibitory synapses. 
Eq. (14) is the expression of $\alpha$ function, and the symbol $\Theta(\mathrm{t})$ represents Heaviside step function. $\tau$ is the characteristic constant of the synapse, when the synapse is excitatory, $\tau=2 \mathrm{~ms}$, and when the synapse is inhibitory, $\tau=$ 4ms. In addition, we use Euler's method to solve the HH equation numerically with a step size of $0.01 \mathrm{~ms}$.

\section{4 power norm}

To intuitively quantify the signal propagation in neural network, the power norm (or fidelity $C_{0}$ ) of signal in output layer is calculated. Power norm can be calculated by the following formula:

$$
C_{0}=\max \left\{\overline{\left(g_{\text {in }}(t)-\overline{g_{\text {in }}(t)}\right)\left(\operatorname{PSTH}\left(t+\tau_{d}\right)-\overline{P S T H(t)}\right)}\right\},
$$

Where, PSTH (t) (post-stimulus time histogram) counts the number of neurons firing accumulated in a bin $(\mathrm{bin}=$ $1 \mathrm{~ms})$. The fidelity $C_{0}$ of signal transmission is measured by comparing the similarity between the signal $g_{i n}(\mathrm{t})$ and the tenth layer response PSTH $\left(t+\tau_{d}\right)_{10}$. In addition, we choose the time lag that maximizes $C_{0}$ as $\tau_{d}$. The power norm $C_{0}$ reflects the accuracy of signal transmission, and the time lag $\tau_{d}$ reflects the speed of signal transmission.
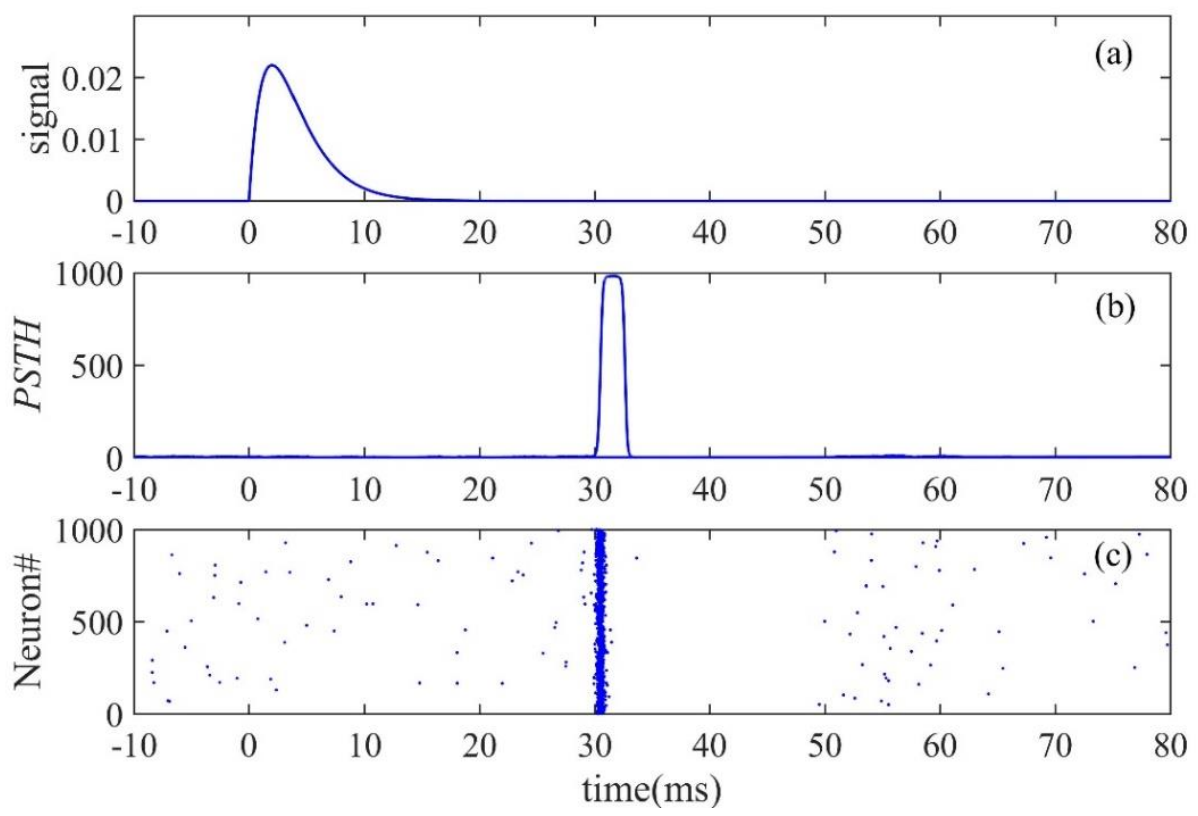

Fig.3 Schematic diagram of power norm; a Time series diagram of signal received by each neuron in the first layer; $\mathbf{b}$ $P S T H$ of the output layer; c Dot raster plot of spike time of the output layer. The power norm (fidelity) $C_{0}$ is obtained by calculating the correlation between input signal and output signal

\section{Numerical results and discussion}

3.1 Influence of temperature and noise intensity on signal transmission

In this section, we study the influence of temperature and noise intensity on signal transmission. Related to the expression of temperature in the mathematical model of neurons, we only take the temperature range of $0^{\circ} \mathrm{C}-14^{\circ} \mathrm{C}$. As 
shown in Fig.4, first fixed temperature $T=9^{\circ} \mathrm{C}$, and increasing the noise intensity from 0 to 3 . When the noise intensity is very small $(D=0.95)$, a weak response to the signal appears in the first layer, then the response becomes weaker in the next layer. In the output layer, there is no tangible response to the signal, indicating that the signal fails to transmit to the output layer. As the noise intensity increases, there is a threshold for the noise intensity, that is, the minimum noise intensity for the signal to propagate successfully. After the noise intensity increases to the threshold $(D=1.05)$, a distinct response to the signal is observed in the output layer and the signal transmits to the output layer successfully. However, further increase in noise intensity will suppress the signal propagation. In a particular point $D=1.4$ (Fig.4c), the pulse packet triggered by the noise meets the signal and interferes with the signal propagation in the output layer. When the noise intensity continues to increase to a large value $(D=2.85)$, it can be seen from Fig.4d that the signal becomes dissipated in the output layer, and the excessive noise induces multiple pulse packets that are even more concentrated than the signal.
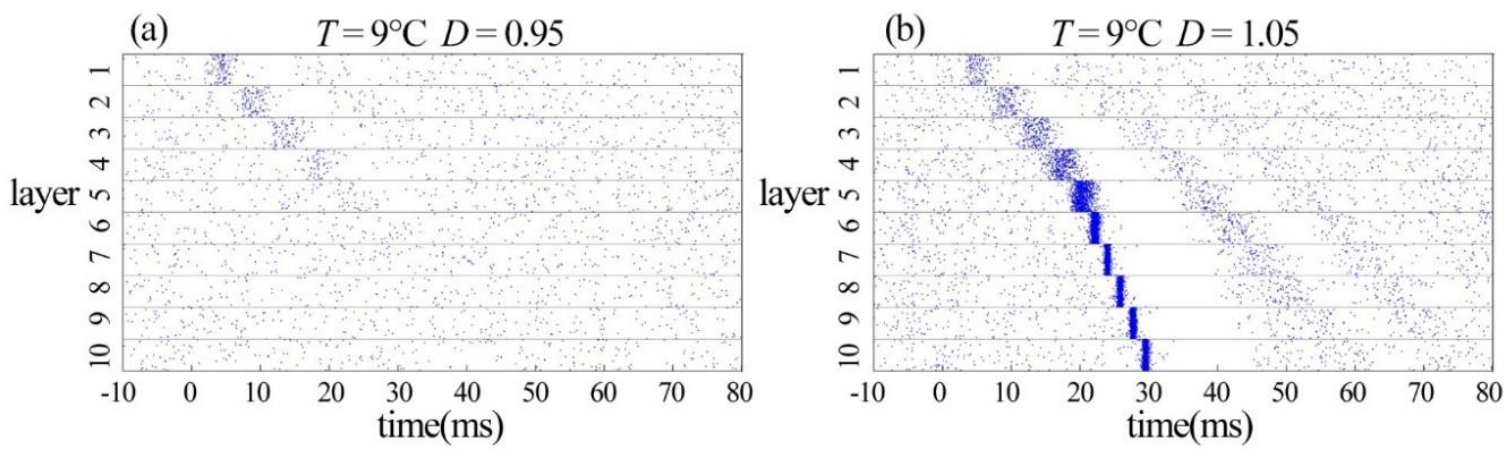

(c) $T=9^{\circ} \mathrm{C} D=1.4$

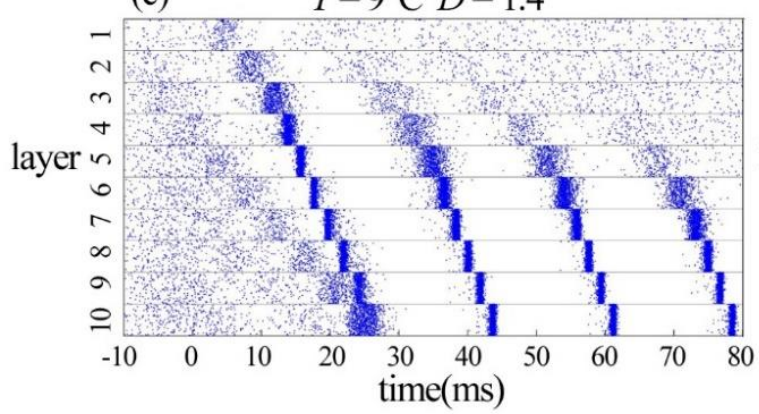

(d)

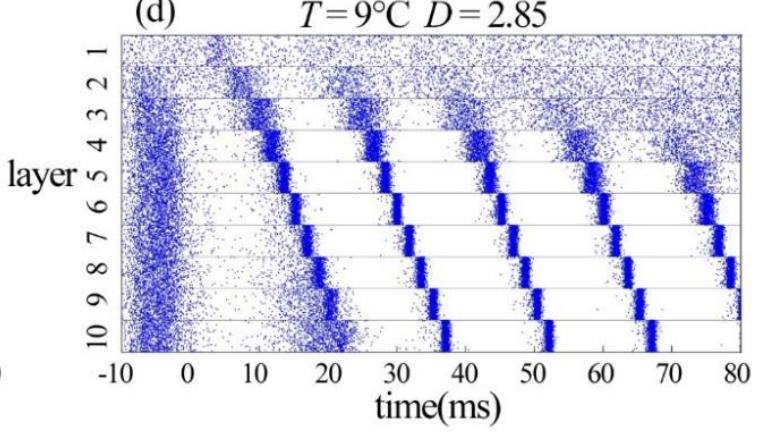

Fig.4 At fixed temperature $T=9^{\circ} \mathrm{C}$, the spatial-temporal diagram of signal propagation in a ten-layer feedforward neural network under different noise intensity; $\mathbf{a} D=0.95 ; \mathbf{b} D=1.05 ; \mathbf{c} D=1.4 ; \mathbf{d} D=2.85$. The noise intensity in a is too small for the signal to propagate to the output layer. The noise intensity in $\mathbf{b}$ is exactly the threshold for successful signal propagation. And the noise intensity in $\mathbf{c}$ and $\mathbf{d}$ is too large, so the noise interferes with the signal propagation

In Fig.5, PSTH of layer 1, 3, 5, 7 and 10 corresponding to the four cases in Fig.4 are plotted. When the noise intensity is large, pulse packets caused by noise confuses the signal in output layer. In order to facilitate the identification of the signal, we mark the signal with arrows of corresponding colors in Fig.5. If the peak that represents 
the signal is narrower and higher, it means that the signal is more concentrated and the signal propagates better. The results obtained by PSTH diagram are consistent with the spatial-temporal diagram. The signal cannot be successfully transmitted to the output layer until the noise intensity is increased to the threshold value, at the threshold value, the signal propagates best. The subsequent increase in noise intensity will interfere with the signal and suppress the signal propagation. The excessive noise will lead to the dissipation of the signal in the output layer and the confusion of the signal by noise.

In addition, it is noticed that when the noise intensity is large $(D=3)$, the temperature has great effects on signal transmission, as shown in Fig.6a, b. At low temperature $\left(T=0.5^{\circ} \mathrm{C}\right)$, the signal will be completely submerged in the irregular network activity caused by noise after being transmitted through several layers, and there are no discernable signal-triggered spikes in the output layer. Raising temperature can enhance the anti-noise ability of the signal propagation. When the temperature is higher $\left(T=13.5^{\circ} \mathrm{C}\right)$, the signal will still be able to transmit to the output layer, but due to the interference of strong noise, the propagation will be worse. Furthermore, we find that the noise-induced pulse packet could not only merge with the signal and weaken the signal propagation, as shown in Fig.4c, but also force the signal to suddenly dissipate so that the signal could not propagate at all, this phenomenon is later referred as the jumping phenomenon. As in Fig.6c, d, when $T=3^{\circ} \mathrm{C}$ and $D=0.95$, the noise-induced pulse packet forces its adjacent signal to dissipate in the layer 9 , so there is no signal-triggered spike in the output layer. Differently, when $T=3^{\circ} \mathrm{C}$ and $D=1$, the signal in the layer 6 becomes weak under the interference of noise, but it does not disappear completely. At the cost of the transmission speed, the weak signal is still transmitted to the output layer. However, the time lag of the signal is very large. In Fig.7, the variation of signal fidelity $C_{0}$ and time lag $\tau_{d}$ with noise intensity $D$ at different temperatures is plotted, and the figure at $T=3^{\circ} \mathrm{C}$ also confirms the jumping phenomenon in the value of $C_{0}$ caused by the noise. The reason may be that the noise-induced pulse packet is too strong and too close to the signal, so the network has no time to respond to the signal. 

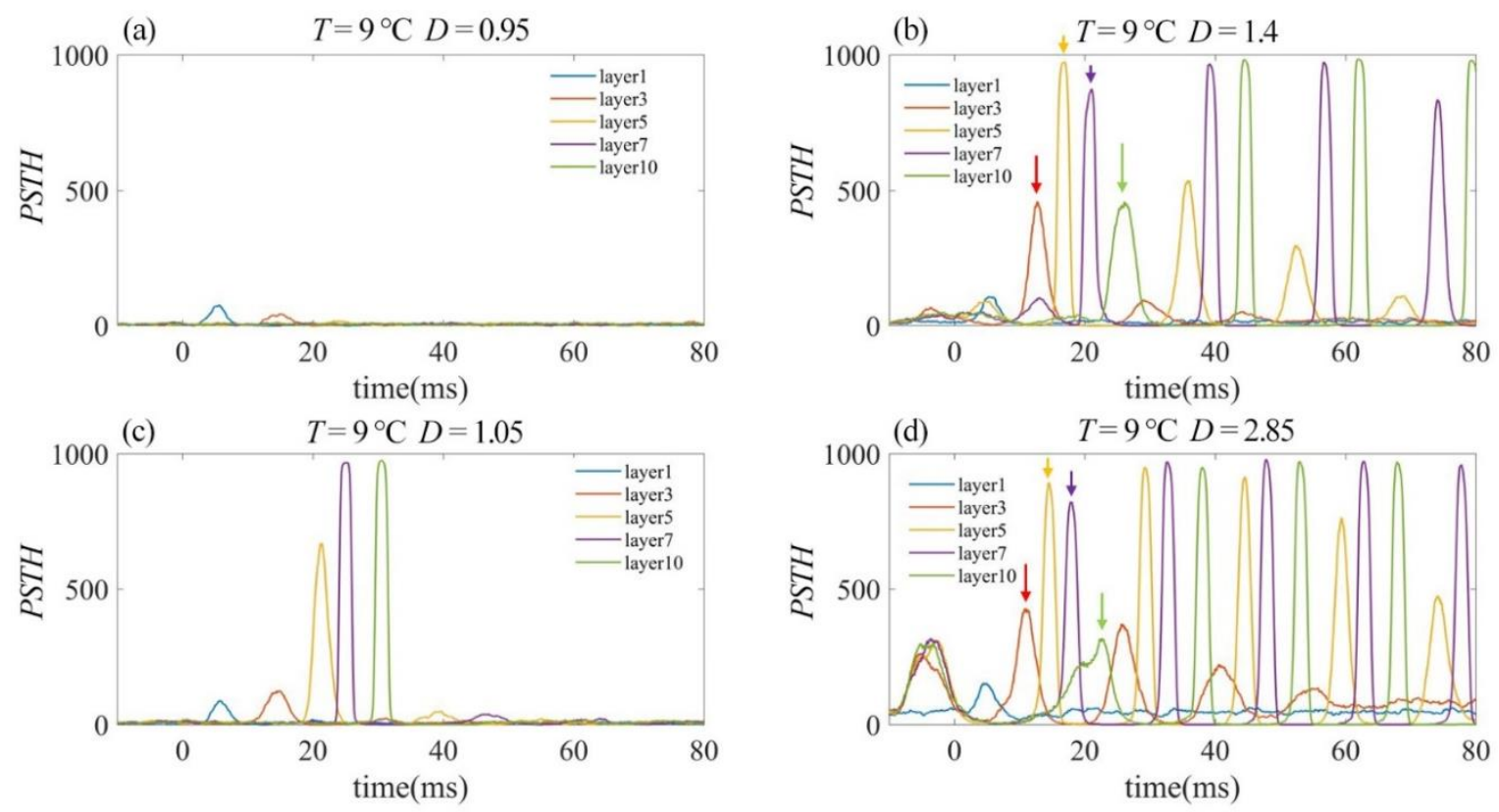

Fig.5 PSTH of layer 1, 3, 5, 7 and 10 networks under different noise intensity at fixed temperature $T=9^{\circ} \mathrm{C} ; \mathbf{a} D=0.95$; b $D=1.4 ; \mathbf{c} D=1.05 ; \mathbf{d} D=2.85 ;$ Under the conditions of $D=1.4$ and $D=2.85$, the peaks that represent signals in layer3, layer5, layer7 and layer10 are marked with arrows of corresponding colors. The results obtained by PSTH figure are consistent with the spatial-temporal diagram
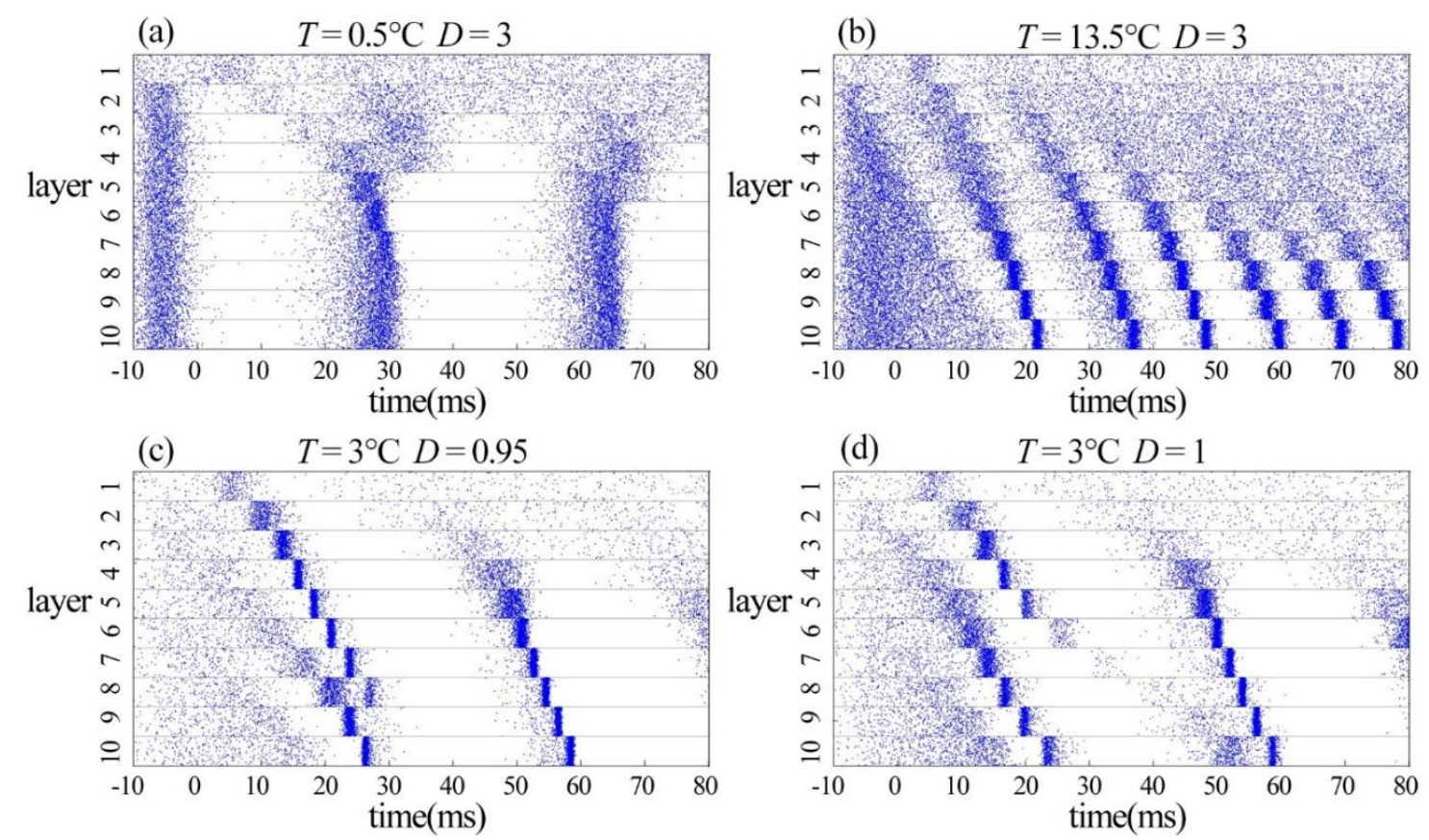

Fig.6 Spatial-temporal diagram of signal propagation for some specific temperature and noise conditions; $\mathbf{a} T=0.5^{\circ} \mathrm{C}$, $D=3 ; \mathbf{b} T=13.5^{\circ} \mathrm{C}, D=3 ; \mathbf{c} T=3^{\circ} \mathrm{C}, D=0.95 ; \mathbf{d} T=3{ }^{\circ} \mathrm{C}, D=1$. From $\mathbf{a}$ and $\mathbf{b}$, it can be seen that under large noise, temperature has a great influence on signal propagation. And the jumping phenomenon appears in $\mathbf{c}$ and $\mathbf{d}$ 
Next, the influence of temperature on signal propagation is concerned. As shown in Fig.7, with the increase of temperature, the noise intensity threshold $D$ required for signal propagation increases gradually, and the value of $C_{0}$ under different $D$ decreases generally. Indicating that the increase in temperature will deteriorate signal propagation, so that the signal propagation requires larger noise intensity.
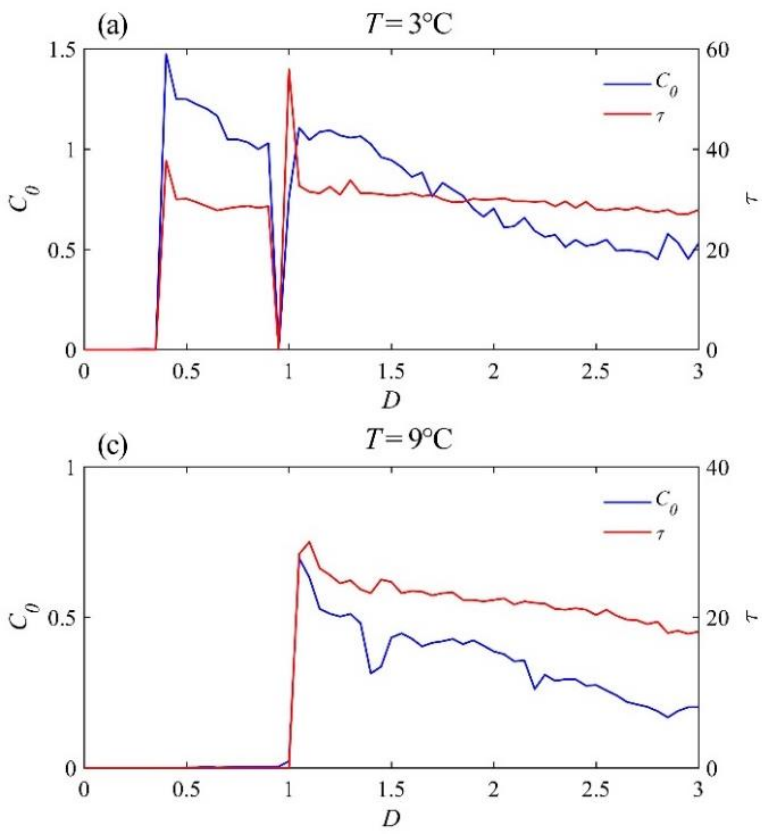
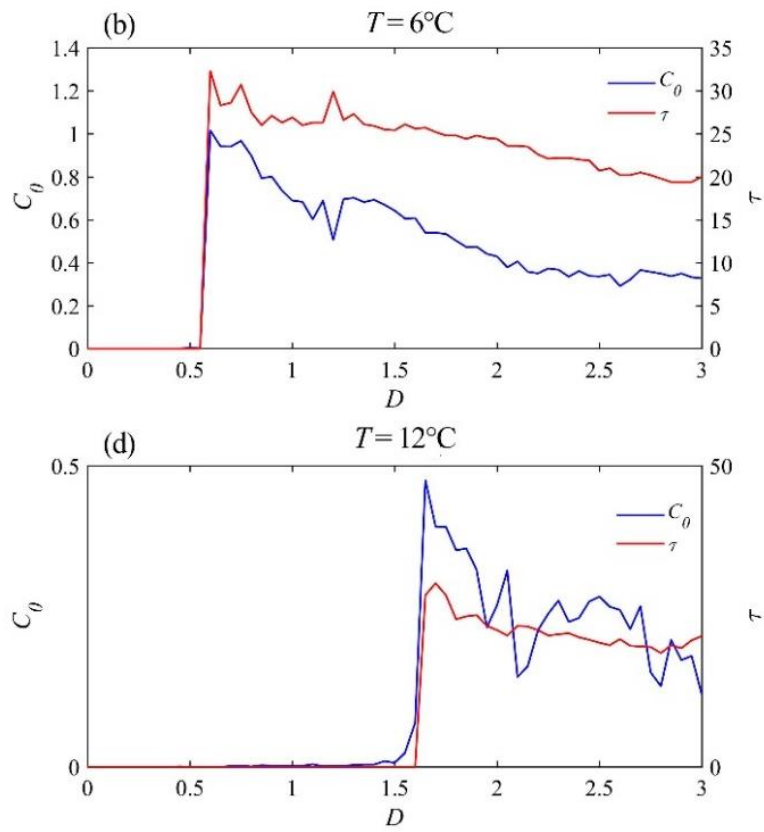

Fig.7 Signal fidelity $C_{0}$ and time lag $\tau_{d}$ in output layer vary with noise intensity $D$ at different temperatures; $\mathbf{a} T=3^{\circ} \mathrm{C}$; b $T=6^{\circ} \mathrm{C} ; \mathbf{c} T=9^{\circ} \mathrm{C} ; \mathbf{d ~} T=12^{\circ} \mathrm{C}$. In figure a, there is a jump in the value of $C_{0}$ and $\tau_{d}$. As the temperature increases, the noise intensity threshold increases, and the fidelity $C_{0}$ decreases generally
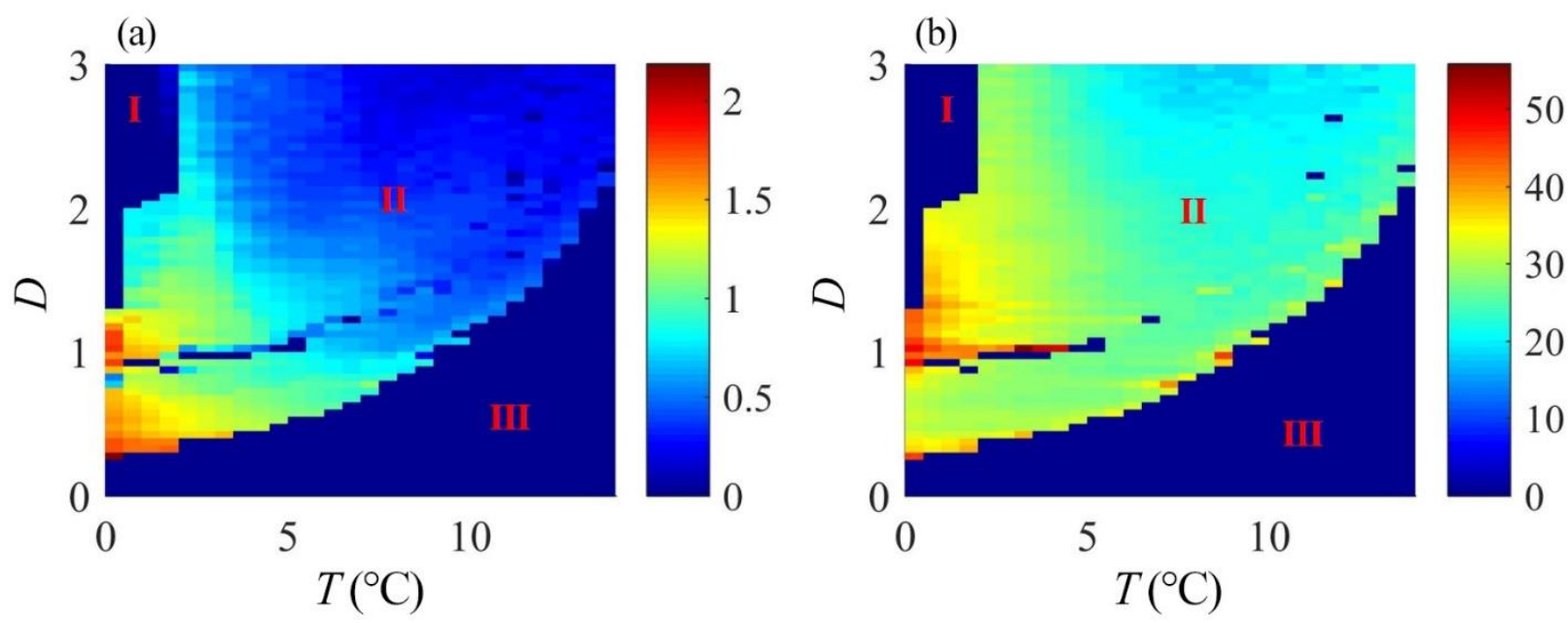

Fig.8 The signal fidelity $C_{0}$ and time lag $\tau_{d}$ depending on noise intensity and temperature; a the color represents the power norm; b the color represents the time lag of signal transmission. The figures are divided into three regions, I and 
III are the region where the signal fails to propagate stably, and II is the region where the signal succeeds to propagate.

Finally, the two two-dimensional diagrams of fidelity $C_{0}$ and time lag $\tau_{d}$ changing with noise intensity and temperature are drawn in Fig.8 respectively. The figures are divided into three regions: I is the region of low temperature and strong noise where the blue color indicates that the signal in this region fails to transmit, II is the region where the signal propagates successfully, and III is the region below the threshold of noise intensity where the signal can't propagate successfully too. We find that it is common for noise to force the signal to dissipate, then resulting in the jumping in the value of signal fidelity $C_{0}$ and time lag $\tau_{d}$, and the blue patches scattered among the colored areas concentrated in $T<8^{\circ} \mathrm{C}$ and $D \cong 1.1$ in Fig.5a, b represent the occurrence of jumping. Similarly, it can be seen from the boundaries of region II and III that the rise in temperature will increase the value of noise intensity threshold. The failure propagation of signal in region I indicates that when the temperature is low $\left(T<2^{\circ} \mathrm{C}\right)$, the anti-noise capability of the signal propagation is weak. Moreover, from Fig.8b, it's found that except for jumping points, the increasing of noise intensity and temperature can speed up the signal propagation, resulting the decrease of time lag $\tau_{d}$. At special jumping points, there is a sudden decrease in the value of fidelity $C_{0}$ and time lag $\tau_{d}$ because of the noise.

\subsection{Effect of interlayer connection probability $P_{\text {inter }}$ on signal transmission}

In this section, the influences of the interlayer connection probability on the signal transmission are discussed in the ten-layer feedforward neural network. It is known that the neuron will only fire when the sum of synaptic currents received from connected neurons exceeds a certain threshold. Therefore, we can reasonably guess when the interlayer connection probability of feedforward network is very small, the signal will propagate badly. Along with the increasing of the interlayer connection probability, the signal propagation will be better. The following content will test the correctness of this conjecture.

In Fig.9, the curves of the signal fidelity $C_{0}$ in output layer changing with the noise intensity $D$ at different temperatures $\left(T=2^{\circ} \mathrm{C}, T=6^{\circ} \mathrm{C}, T=8^{\circ} \mathrm{C}, T=10^{\circ} \mathrm{C}, T=12^{\circ} \mathrm{C}, T=14^{\circ} \mathrm{C}\right)$ and at different interlayer connection probabilities $\left(P_{\text {inter }}=0.001, P_{\text {inter }}=0.008, P_{\text {inter }}=0.05, P_{\text {inter }}=0.1\right)$ are plotted. Under the condition that $T<14^{\circ} \mathrm{C}$, when the connection probability is too small $P_{\text {inter }}=0.001$, no matter what the temperature is, $C_{0}$ is very small, indicating that neurons have not received enough input to fire. When $P_{\text {inter }}$ increases to 0.008 and the noise intensity increases to the threshold value, the fidelity $C_{0}$ obviously transitions to a larger value. As the noise intensity $D$ continues to increase, $C_{0}$ will gradually decrease and the jump phenomenon caused by noise will appear, which is similar to the result in Section 3.1. When the interlayer connection probability increases to $P_{\text {inter }}=0.05$, the noise intensity threshold where the failed signal propagation transforms into succeed signal propagation increases. When the interlayer connection 
probability continues to increase to $P_{\text {inter }}=0.1$, the curve obtained by $P_{\text {inter }}=0.1$ is similar to the curve obtained by $P_{\text {inter }}$ $=0.05$, or even partially overlapped. It indicates that when the interlayer connection probability exceeds $P_{\text {inter }}=0.05$, the signal propagation is basically unchanged as the $P_{\text {inter }}$ continues to increase. It can be proposed that $P_{\text {inter }}=0.05$ is the saturation value of the interlayer connection probability. Surprisingly, such a small interlayer connection probability is sufficient for the signal to propagate successfully, and the larger interlayer connection probability cannot improve the signal transmission. The too large interlayer connection probability is a waste of energy, and the appropriate interlayer connection probability makes signal transmission more efficient. In the case of excessively high temperature $T=14^{\circ} \mathrm{C}$, it can still be found that the increase in interlayer connection probability will lead to the increase in noise intensity threshold. However, the large randomness brought by strong noise will lead to the irregular propagation of signal, as a result, the value of $C_{0}$ has large fluctuations.

In addition, from a lateral point of view, it's found that no matter what the interlayer connection probability is, increasing temperature will lead to an increase in the noise intensity threshold and a decrease in $C_{0}$ value. This shows that the effect of temperature on signal propagation in FFN is independent of the interlayer connection probability.
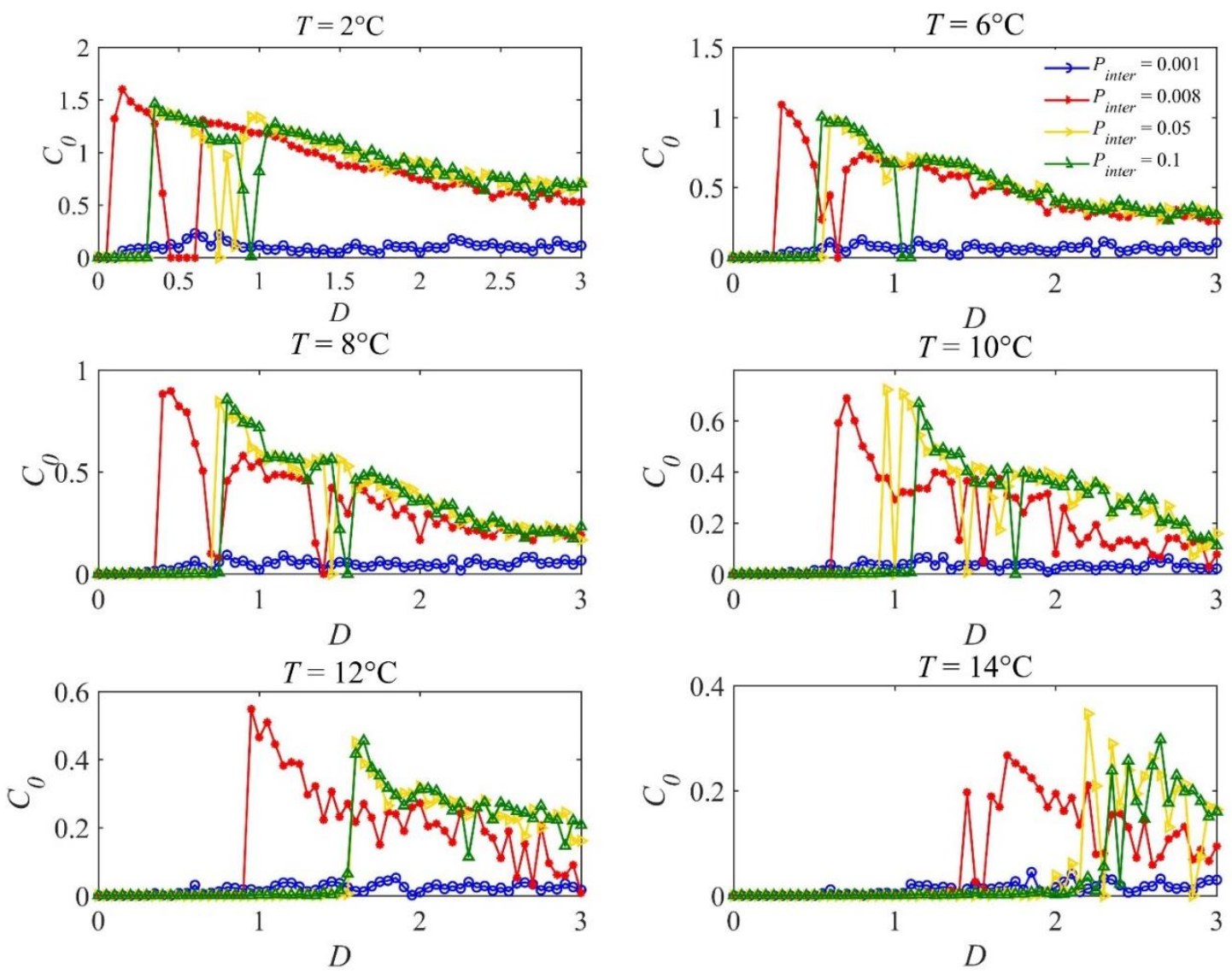

Fig.9 The curve of signal fidelity $C_{0}$ in the output layer changing with noise intensity $D$ at different temperatures and 
different interlayer connection probabilities. The colors corresponding to different connection probabilities are: blue for $P_{\text {inter }}=0.001$, red for $P_{\text {inter }}=0.008$, yellow for $P_{\text {inter }}=0.05$, green for $P_{\text {inter }}=0.1 ; \mathbf{a} T=2^{\circ} \mathrm{C} ; \mathbf{b} T=6^{\circ} \mathrm{C} ; \mathbf{c} T=8^{\circ} \mathrm{C}$; d $T=10^{\circ} \mathrm{C} ; \mathbf{e} T=12^{\circ} \mathrm{C} ; \mathbf{f} T=14^{\circ} \mathrm{C}$

\subsection{Signal propagation in EI networks}

In this section, the feedforward network with two kinds of neurons, namely, excitatory and inhibitory neurons is constructed to study the influence of networks connection structure on signal propagation. The EI neural network is constructed in the tight intralayer connection and sparse interlayer connection way, and the connection parameters are given in section 3.2. As stated in the introduction, in EI neural networks, because of the interaction between excitatory and inhibitory neurons, the network can generate different kinds of spontaneous activity. The imbalance of EI causes the neural network to oscillate and synchronize. However, the balance of EI will cancel out their effect, leading to the neurons fire randomly and irregularly. In this section, in order to eliminate the interference of the imbalanced strength of EI neurons, the strength of inhibitory synapses and excitatory synapses is fixed to be the same. We mainly concentrate on the relationship between the spontaneous activity of the EI network and the signal propagation, and the differences between signal propagation in EI network and pure excitatory network under varied temperature and noise intensity.
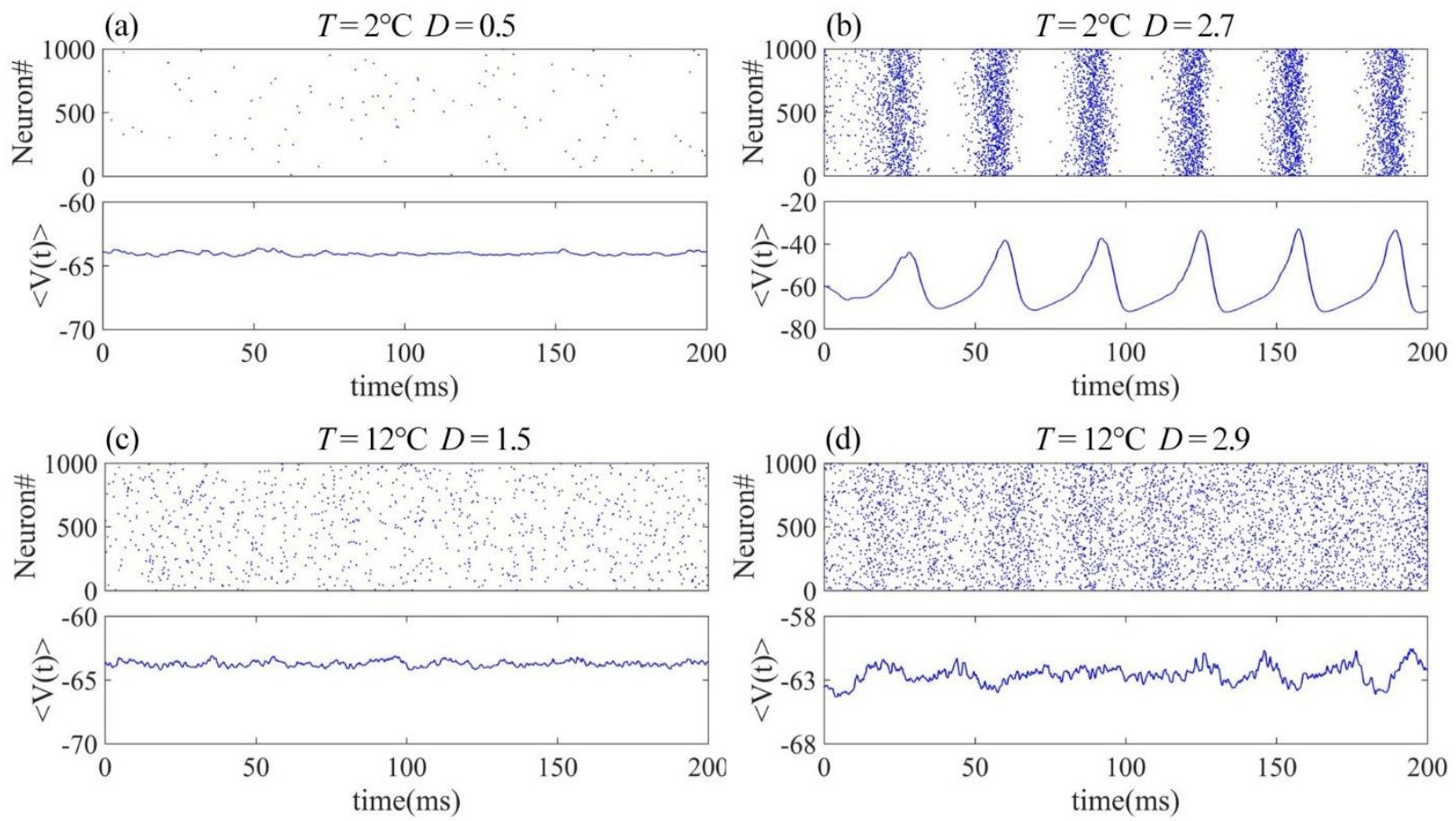

Fig.10 Spontaneous activity of an isolated EI neural network under different noise intensity and temperature. Interlayer connection probability is $P_{\text {inter }}=0.05 ; \mathbf{a} T=2{ }^{\circ} \mathrm{C}, D=0.5 ; \mathbf{b} T=2{ }^{\circ} \mathrm{C}, D=2.7 ; \mathbf{c} T=12^{\circ} \mathrm{C}, D=1.5 ; \mathbf{d} T=12^{\circ} \mathrm{C}, D=$ 
2.9. $\mathbf{b}$ is in the synchronous regular (SR) state; $\mathbf{d}$ shows the tendency of synchronous regular (SR) state; $\mathbf{a}$ and $\mathbf{c}$ are in the asynchronous irregular (AI) state. The increase in noise intensity and the decrease in temperature tend to transform the network from AI state to SR state

Firstly, we study the influence of noise intensity $D$ and temperature $T$ on the spontaneous activity of an isolated EI neural network without the external input signal. It can be seen from Fig.10 that when both temperature and noise intensity are small $\left(T=2^{\circ} \mathrm{C}, D=0.5\right)$, the firing of neurons is irregular and the correlation between neurons is small, thus EI network is in the AI state. When we fix temperature $T=2^{\circ} \mathrm{C}$ and the noise intensity increases to $D=2.9$, there will exist rhythmic oscillation in the EI network, which means that the EI network is in the SR state. In Fig.10c, when $T=12^{\circ} \mathrm{C}$ and $D=1.5$, the EI network is in the AI state with more irregular spikes. When fixing $T=12^{\circ} \mathrm{C}$ and the noise intensity increases to $D=2.9$, the neurons in EI network fire more actively and show the trend of synchronization due to the increase of the noise. In conclusion, the increase in noise intensity $D$ will transform the EI network from AI state to SR state, while the increase in temperature can inhibit this transition.

The signal propagation in 10-layer EI network corresponding the above four situation is shown in Fig.11, where the interlayer connection probability is $P_{\text {inter }}=0.05$. We attempt to explore the relationship between the intrinsic activity of the isolated EI network and the signal transmission. It's found that when the temperature and noise is small $\left(T=2^{\circ} \mathrm{C}\right.$, $D=0.5)$, and the EI network is in AI state, the signal can propagate stably to the output layer. When the EI network is in SR state $\left(T=2^{\circ} \mathrm{C}, D=2.9\right)$, the signal cannot be distinguished from the spontaneous oscillation of the network in the first layer, so that the signal is submerged in it and cannot be transmitted to the output layer. Similarly, when the temperature is high $\left(T=12^{\circ} \mathrm{C}\right)$, and the noise intensity is moderate $(D=1.5)$, the EI network is in the AI state, thus the signal succeeds to stably propagate to the output layer. When the noise intensity is high $(D=2.9)$, the neurons in EI network tend to synchronous discharge. The excessively active spontaneous activity and the tendency to synchronize in the EI network caused by noise make it difficult for the signal to propagate to the output layer.

Next, the curve of the signal fidelity $C_{0}$ in output layer changing with the noise intensity $D$ at different temperatures are plotted, as shown in Fig.12. Different from Fig.7, no matter what the temperature is, when the noise intensity is too large, the signal will be completely submerged in the spontaneous oscillation activity of the EI network in the first layer, resulting in $C_{0}$ falling to 0 . While in the pure excitatory network, when the temperature is large enough the signal can still propagate under the strong noise. In addition, the noise intensity threshold where the failed signal propagation turns to successful propagation in EI network is lower than the noise intensity threshold in pure excitatory networks. From Fig.12, it can be seen that there also exists jumping phenomenon caused by the noise in the value of fidelity $C_{0}$ 
in the EI network.

Finally, the two-dimensional graph of fidelity $C_{0}$ changing with noise intensity and temperature is drawn in Fig.13a. By comparing Fig.8 and Fig.13a, it is found that in the EI network, the parameter region where the signal can successfully propagate is smaller, while the region where the signal fails to successfully propagate is larger, and the fidelity $C_{0}$ in the EI network is smaller on the whole. The reason may be that inhibitory neurons in EI networks inhibit neuronal firing, making signal transmission more difficult than in pure excitatory networks. As shown in Fig.13, when the temperature is fixed, as the noise intensity increases, the value of $C_{0}$ increases from 0 to non- 0 and then decreases to 0 , corresponding to three signal propagation state, that is, failed propagation $\rightarrow$ successful propagation $\rightarrow$ failed propagation. With the increase of temperature, the noise intensity threshold increases, and the range of noise intensity for which the signal propagates successfully becomes wider, but the $C_{0}$ value becomes smaller. The two-dimensional graph of fidelity $C_{0}$ under the situation of interlayer connection probability $P_{\text {inter }}=0.03$ is also plotted in Fig. $13 \mathrm{~b}$, which is similar to Fig.13a.
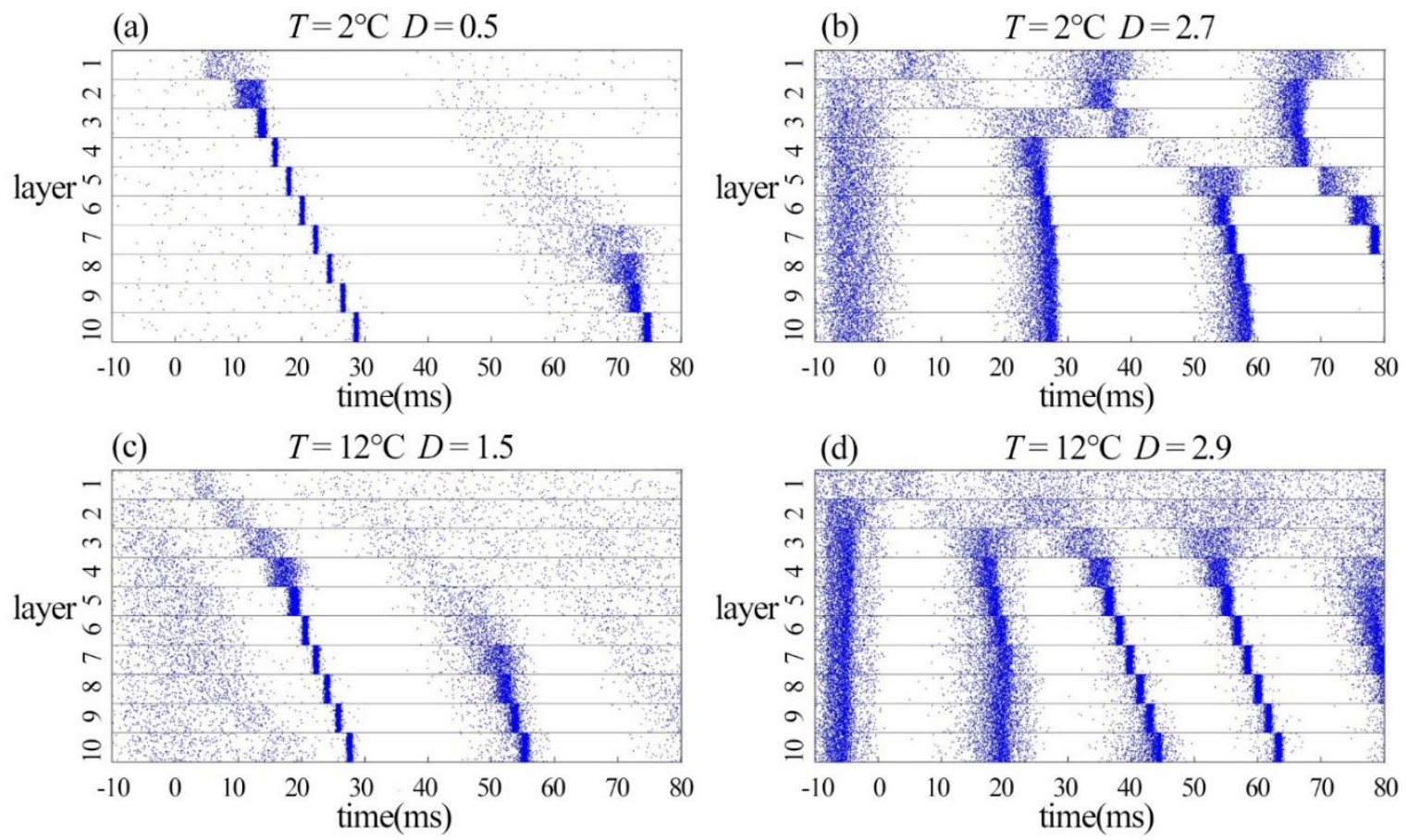

Fig.11 Spatial-temporal diagram of signal propagation in the 10-layer EI network for the four cases. $\mathbf{a}=12^{\circ} \mathrm{C}, D=$ $0.5 ; \mathbf{b} T=2{ }^{\circ} \mathrm{C}, D=2.7 ; \mathbf{c} T=12^{\circ} \mathrm{C}, D=1.5 ; \mathbf{d} T=12^{\circ} \mathrm{C}, D=2.9$. In $\mathbf{a}$ and $\mathbf{c}$, when the spontaneous activity of the EI network is AI state, the signal propagates stably to the output layer. In $\mathbf{b}$ and $\mathbf{d}$, when the spontaneous activity of EI network is SR or shows the trend of SR, the signal will be covered by the network activity, and fails to propagate. The interlayer connection probability $P_{\text {inter }}=0.05$ 

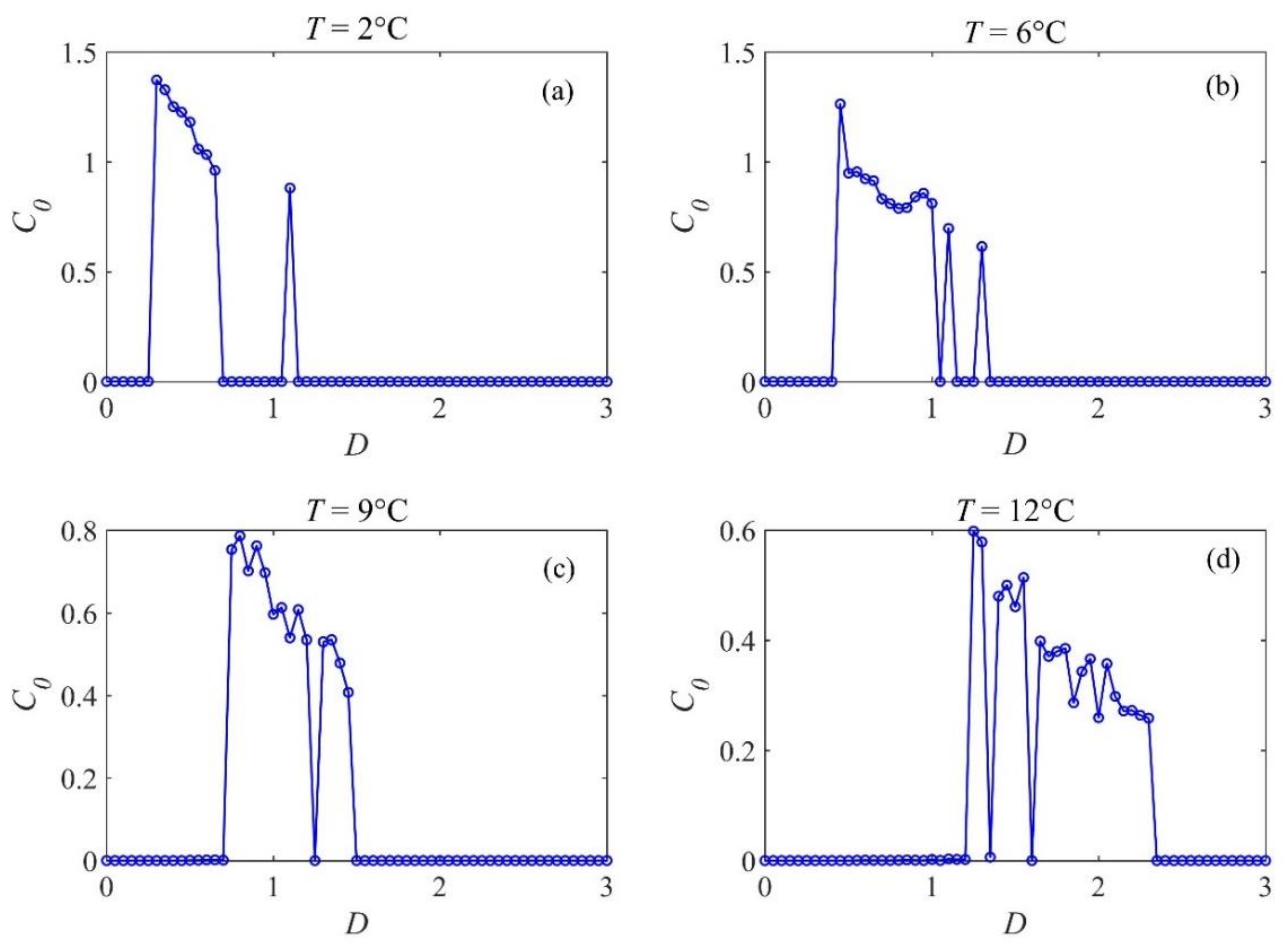

Fig.12 Curve of signal fidelity $C_{0}$ in the output layer changing with noise intensity $D$ at different temperatures; $\mathbf{a} T=$ $3^{\circ} \mathrm{C} ; \mathbf{b} T=6^{\circ} \mathrm{C} ; \mathbf{c} T=9^{\circ} \mathrm{C} ; \mathbf{d} T=12^{\circ} \mathrm{C}$. As the temperature increases, the range of noise intensity where the signal can successfully propagate shifts to the right. At all temperatures, when the noise intensity is too small or too large, the signal fails to propagate. The jumping phenomenon in the value of fidelity $C_{0}$ appears. The interlayer connection probability $P_{\text {inter }}=0.05$

In conclusion, in addition to noise intensity and temperature, the connection structure of FFN also has a great influence on signal propagation. When the neural network is purely excitatory, there is no intralayer connection between the neurons in the network, and there is no spontaneous activity of synchronous oscillations. However, in EI feedforward neural network, the interactions between excitatory neurons and inhibitory neurons enable the isolated neural network show different spontaneous activity. When the EI neural network is in the state of AI with moderate noise, the signal propagates reliably. When the EI neural network is in the state of SR with the large noise, the signal cannot propagate to the output layer. What needs to be distinguished is that in pure excitatory network, under low temperature and large noise intensity, the signal is also submerged in the noise-induced network activity in deeper layers. In EI network, at all temperatures and under strong noise, the neural network will show the intrinsic activity of synchronous oscillation, or the trend of synchronous oscillation, which will cover the signal in the first layer. But, in the pure excitatory network, there is no such intrinsic activity of synchronous oscillation. 

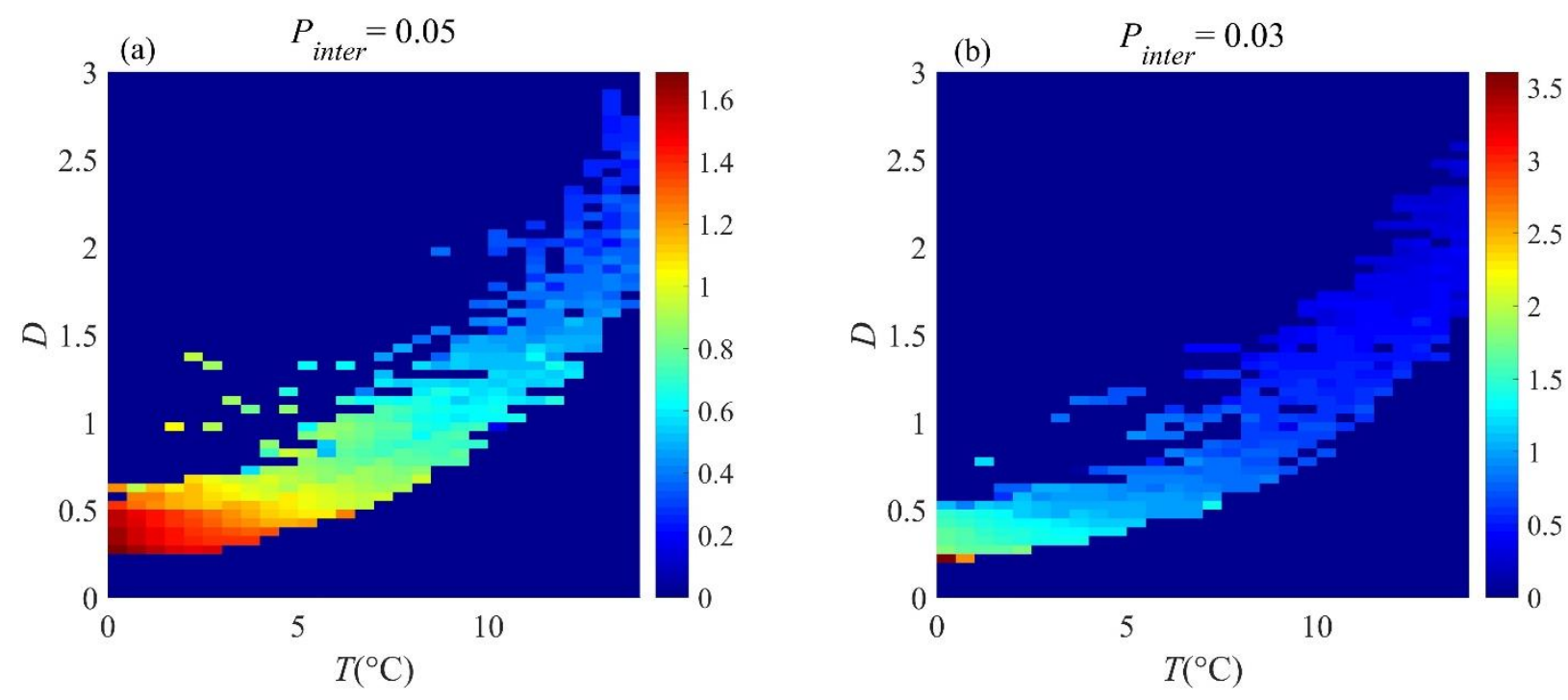

Fig.13 The variation of signal fidelity $C_{0}$ in output layer with noise intensity $D$ and temperature $T$ in EI network; (a) the interlayer connection probability $P_{\text {inter }}=0.05$. (b) the interlayer connection probability $P_{\text {inter }}=0.03$. Compared with the two-dimensional graph in pure excitatory network, the region where the signal propagates successfully in EI network is smaller.

\section{Conclusions and discussions}

It has been revealed that temperature is of functional importance in regulating neuron dynamics recently, however, the previous studies are mainly based on constant temperature $T=6.3^{\circ} \mathrm{C}$ [52]. In this paper, by introducing the influence of temperature on neuron membrane ion channel in the neuron model, the effect of temperature on subthreshold signal propagation in the ten-layer feedforward network is considered. Moreover, the effects of noise intensity, interlayer connection probability and network's connection structure on signal propagation are also investigated. The subthreshold signal injected in the input layer is in the form of EPSC (excitatory post-synaptic current), which is the functional simulation of the actual synaptic current. The power norm $C_{0}$ is calculated to measure the fidelity between the system's response and the subthreshold EPSC signal, that is obtained by comparing the similarity between the input signal and the output signal. The spatial-temporal diagram of signal propagation and the PSTH of network in layer 1, layer3, layer 5, layer7 and layer10 are plotted.

On the one part, for the pure excitatory ten-layer feedforward neural network, the effects of temperature, noise intensity, and interlayer connection probability on the signal propagation are investigated. The main results can be summarized in the following three points:

(i) Under the constant temperature, as the increase of noise intensity, the signal propagation undergoes three 
processes. When the noise intensity is smaller than the certain value, the signal fails to propagate to the output layer. After the noise intensity reaches the certain value, the failed signal propagation can transform into succeed signal propagation. Finally, the further increase of noise intensity can weaken the signal propagation. Except for these three signal propagation processes, some specific results are concluded. Under the large noise, the signal propagation in different temperatures exhibits different antinoise capabilities. That is, the signal will be submerges in the noise-triggered network activity completely in the low temperature. In the high temperature, the signal still propagates successfully to the output layer.

(ii) The increase of temperature can raise the noise intensity threshold, which is the certain value where the failed signal propagation turns to successful propagation. Moreover, increasing temperature can worsen the signal propagation, resulting in the decrease in fidelity $C_{0}$.

(iii) The signal propagation is terrible with too small interlayer connection probability $P_{\text {inter }}=0.001$. Increasing the interlayer connection probability can raise the noise intensity threshold until the $P_{\text {inter }}$ reaches the saturation value $\left(P_{\text {inter }}=0.05\right)$. If the connection probability is greater than saturation value $\left(P_{\text {inter }}=0.05\right)$, the fidelity and signal propagation state remain unchanged. Moreover, the influence of temperature on signal propagation is independent of the connection probability.

(iv) It's known that signals tend to be gradually synchronized in the transmission between layers because different neurons share a common input, but this tendency will be disturbed by noise, leading to the jumping phenomenon in the value of fidelity $C_{0}$. The noise-triggered spikes form a pulse packet after several layers, and this pulse packet conflicts with the signal, destroying the signal propagation, therefore, the strong noise destroys signal stable propagation.

On the other part, for the ten-layer EI feedforward neural network, the influence of network structure, temperature, and noise intensity on subthreshold signal transmission are studied. We mainly focus on two issues, one is the relationship between spontaneous activities and signal propagation in EI network, the other is the differences between signal propagation in the EI network and signal propagation in the pure excitatory network. The results can be summarized as following:

(i) The interaction between excitatory and inhibitory neurons in the isolated EI network enables the network show complex spontaneous activity, i.e., synchronous regular state (SR) and asynchronous irregular state (AI). The increase in noise intensity can transform the EI network from AI state to SR state, and the 
increase in temperature inhibits this transition. When the EI network is in the state of AI, the signal succeeds to propagate to the output layer; When the EI network is in the state of SR, the synchronous oscillation of EI network will cover the subthreshold signal in the first layer, thus destroying the signal propagation.

(ii) The region where the subthreshold signal succeeds to propagate on the plane of temperature and noise intensity parameters in the EI network is smaller than the region in the pure excitatory network. The region's reduction is caused by the reason that the SR spontaneous activity induced by large noise in EI network can inhibit signal propagation. In addition, under the same temperature, the noise intensity threshold in EI network is smaller than the threshold in pure excitatory network.

(iii) The common characteristics of signal propagation in the EI network and signal propagation in the pure excitatory network are: the increase in temperature can raise the noise intensity threshold, and the jumping in the value of signal fidelity $C_{0}$ appears in both two type networks.

This work has investigated the temperature effect on signal transmission in feedforward neural network. Next, a more realistic neuron model considering temperature can be selected for simulation. In addition, the connections between neural networks are not purely feedforward connection. There are a small number of feedback connections, which can produce the reverberation between the layers, thus amplifying the signal and facilitating the propagation of the signal[53,54]. So, a more real feedforward neural network with feedback connections can be used to study the signal propagation.

Acknowledgements This work was supported by the National Natural Science Foundation of China under Grants Nos. 11675060, 11772306, and 12172340, and supported by Shanghai Municipal Science and Technology Major Project (No.2018SHZDZX01), Key Laboratory of Computational Neuroscience and Brain-Inspired Intelligence (LCNBI) and ZJLab".

Data availability The datasets generated during and/or analyzed during the current study are available from the corresponding author on reasonable request.

\section{Declarations}

Conflict of interest The authors declare that they have no conflict of interest. 


\section{References}

1. Zhao, Y.,Boulant, J. A.: Temperature effects on neuronal membrane potentials and inward currents in rat hypothalamic tissue slices. J Physiol 564 (Pt 1), 245-257 (2005).

2. Hyun, N. G., Hyun, K. H., Lee, K.,Kaang, B. K.: Temperature dependence of action potential parameters in Aplysia neurons. Neurosignals 20 (4), 252-264 (2012).

3. Pekala, D., Szkudlarek, H.,Raastad, M.: Typical gray matter axons in mammalian brain fail to conduct action potentials faithfully at fever-like temperatures. Physiol Rep 4 (19) (2016).

4. Ramalingam, N.,Dettmer, U.: Temperature is a key determinant of alpha- and beta-synuclein membrane interactions in neurons. J Biol Chem 296, 100271 (2021).

5. Van Hook, M. J.: Temperature effects on synaptic transmission and neuronal function in the visual thalamus. PLoS One 15 (4), e0232451 (2020).

6. Kim, J. A.,Connors, B. W.: High temperatures alter physiological properties of pyramidal cells and inhibitory interneurons in hippocampus. Front Cell Neurosci 6, 27 (2012).

7. Arendt, T., Stieler, J.,Holzer, M.: Brain hypometabolism triggers PHF-like phosphorylation of tau, a major hallmark of Alzheimer's disease pathology. J Neural Transm (Vienna) 122 (4), 531-539 (2015).

8. Lu, L., Kirunda, J. B., Xu, Y., Kang, W., Ye, R., Zhan, X.,Jia, Y.: Effects of temperature and electromagnetic induction on action potential of Hodgkin-Huxley model. The European Physical Journal Special Topics 227 (79), 767-776 (2018).

9. Xing, M., Song, X., Yang, Z.,Chen, Y.: Bifurcations and excitability in the temperature-sensitive Morris-Lecar neuron. Nonlinear Dynamics 100 (3), 2687-2698 (2020).

10. Ding, Q.Jia, Y.: Effects of temperature and ion channel blocks on propagation of action potential in myelinated axons. Chaos 31 (5), 053102 (2021).

11. Xu, Y., Guo, Y., Ren, G.,Ma, J.: Dynamics and stochastic resonance in a thermosensitive neuron. Applied Mathematics and Computation 385 (2020).

12. Song, X., Wang, H., Chen, Y.,Lai, Y. C.: Emergence of an optimal temperature in action-potential propagation through myelinated axons. Phys Rev E 100 (3-1), 032416 (2019).

13. Huh, J. H.: Inverse stochastic resonance in electroconvection by multiplicative colored noise. Phys Rev E 94 (51), 052702 (2016).

14. Lu, L., Jia, Y., Ge, M., Xu, Y.,Li, A.: Inverse stochastic resonance in Hodgkin-Huxley neural system driven by Gaussian and non-Gaussian colored noises. Nonlinear Dynamics 100 (1), 877-889 (2020).

15. Yu, Y., Wang, W., Wang, J.,Liu, F.: Resonance-enhanced signal detection and transduction in the HodgkinHuxley neuronal systems. Phys Rev E Stat Nonlin Soft Matter Phys 63 (2 Pt 1), 021907 (2001).

16. Liu, C., Wang, J., Yu, H., Deng, B., Tsang, K. M., Chan, W. L.,Wong, Y. K.: The effects of time delay on the stochastic resonance in feed-forward-loop neuronal network motifs. Communications in Nonlinear Science and Numerical Simulation 19 (4), 1088-1096 (2014).

17. Collins, J. J., Chow, C. C.,Imhoff, T. T.: Stochastic resonance without tuning. Nature 376 (6537), 236-238 (1995).

18. Yilmaz, E., Baysal, V., Ozer, M.,Perc, M.: Autaptic pacemaker mediated propagation of weak rhythmic activity across small-world neuronal networks. Physica A: Statistical Mechanics and its Applications 444, 538-546 (2016).

19. Ge, M., Wang, G.,Jia, Y.: Influence of the Gaussian colored noise and electromagnetic radiation on the propagation of subthreshold signals in feedforward neural networks. Science China Technological Sciences 64 (4), 847-857 (2020).

20. Wang, G., Ge, M., Lu, L., Jia, Y.,Zhao, Y.: Study on propagation efficiency and fidelity of subthreshold signal in feed-forward hybrid neural network under electromagnetic radiation. Nonlinear Dynamics 103 (3), $2627-2643$ 
(2021).

21. Guo, Y., Zhou, P., Yao, Z.,Ma, J.: Biophysical mechanism of signal encoding in an auditory neuron. Nonlinear Dynamics (2021).

22. Lu, L., Jia, Y., Kirunda, J. B., Xu, Y., Ge, M., Pei, Q.,Yang, L.: Effects of noise and synaptic weight on propagation of subthreshold excitatory postsynaptic current signal in a feed-forward neural network. Nonlinear Dynamics 95 (2), 1673-1686 (2018).

23. Shadlen, M. N.,Newsome, W. T. J. C. O. i. N.: Noise, neural codes and cortical organization. 4 (4), 569-579 (1994).

24. Barral, J., Wang, X. J.,Reyes, A. D.: Propagation of spike timing and firing rate in feedforward networks reconstituted in vitro. (2017).

25. Jacques, Gautrais, and, Simon,Biosystems, T. J.: Rate coding versus temporal order coding: a theoretical approach. 48 (1-3), 57-65 (1998).

26. Zhu, F., Wang, R., Aihara, K.,Pan, X.: Energy-efficient firing patterns with sparse bursts in the Chay neuron model. Nonlinear Dynamics 100 (3), 2657-2672 (2020).

27. Felleman, D. J.,Van, E. J. C. C.: Distributed Hierarchical Processing in the Primate Cerebral Cortex. (1), 1-47 (1991).

28. Ge, M., Jia, Y., Lu, L., Xu, Y., Wang, H.,Zhao, Y.: Propagation characteristics of weak signal in feedforward Izhikevich neural networks. Nonlinear Dynamics 99 (3), 2355-2367 (2019).

29. Lee, A. K.,Wilson, M. A. J. N.: Memory of sequential experience in the hippocampus during slow wave sleep. 36 (6), 1183-1194 (2002).

30. Mehta, M. R., Quirk, M. C.,Wilson, M. A. J. N.: Experience-dependent asymmetric shape of hippocampal receptive fields. 25 (3), 707-715 (2000).

31. Lv, X., Li, X., Cao, J.,Duan, P.: Exponential Synchronization of Neural Networks via Feedback Control in Complex Environment. Complexity 2018, 1-13 (2018).

32. Hu, J., Sui, G., Lv, X.,Li, X.: Fixed-time control of delayed neural networks with impulsive perturbations. Nonlinear Analysis: Modelling and Control 23 (6), 904-920 (2018).

33. Ozer, M., Perc, M., Uzuntarla, M.,Koklukaya, E.: Weak signal propagation through noisy feedforward neuronal networks. Neuroreport 21 (5), 338-343 (2010).

34. Zhao, J., Qin, Y. M.,Che, Y. Q.: Effects of topologies on signal propagation in feedforward networks. Chaos 28 (1), 013117 (2018).

35. Yu, L., Shen, Z., Wang, C., Yu, Y.: Efficient Coding and Energy Efficiency Are Promoted by Balanced Excitatory and Inhibitory Synaptic Currents in Neuronal Network. Front Cell Neurosci 12, 123 (2018).

36. Callaway, E. M.: Feedforward, feedback and inhibitory connections in primate visual cortex. Neural Netw 17 (56), 625-632 (2004).

37. Xu, X., Olivas, N. D., Ikrar, T., Peng, T., Holmes, T. C., Nie, Q.,Shi, Y.: Primary visual cortex shows laminarspecific and balanced circuit organization of excitatory and inhibitory synaptic connectivity. J Physiol 594 (7), 1891-1910 (2016).

38. Salin, P. A.,Bullier, J.: Corticocortical connections in the visual system: structure and function. Physiol Rev 75 (1), 107-154 (1995).

39. Tao, H. W., Li, Y. T.,Zhang, L. I.: Formation of excitation-inhibition balance: inhibition listens and changes its tune. Trends Neurosci 37 (10), 528-530 (2014).

40. Guo, X., Yu, H., Wang, J., Liu, J., Cao, Y.,Deng, B.: Local excitation-inhibition ratio for synfire chain propagation in feed-forward neuronal networks. Physica A: Statistical Mechanics and its Applications 482, 308316 (2017). 
41. Chin, B., Engelman, H.,Scheiffele, P. J. S.: Control of Excitatory and Inhibitory Synapse Formation by Neuroligins. 307 (5713), 1324-1328 (2005).

42. Ma, J.,Tang, J.: A review for dynamics in neuron and neuronal network. Nonlinear Dynamics 89 (3), $1569-1578$ (2017).

43. Hahn, G., Ponce-Alvarez, A., Deco, G., Aertsen, A.,Kumar, A.: Portraits of communication in neuronal networks. Nat Rev Neurosci 20 (2), 117-127 (2019).

44. Hahn, G., Bujan, A. F., Fregnac, Y., Aertsen, A.,Kumar, A.: Communication through resonance in spiking neuronal networks. PLoS Comput Biol 10 (8), e1003811 (2014).

45. Yu, H., Li, K., Guo, X.,Wang, J.: Resonance transmission of multiple independent signals in cortical networks. Neurocomputing 377, 130-144 (2020).

46. Xu, Y., Jia, Y., Ge, M., Lu, L., Yang, L.,Zhan, X.: Effects of ion channel blocks on electrical activity of stochastic Hodgkin-Huxley neural network under electromagnetic induction. Neurocomputing 283, 196-204 (2018).

47. Fu, X.,Yu, Y.: Reliable and efficient processing of sensory information at body temperature by rodent cortical neurons. Nonlinear Dynamics 98 (1), 215-231 (2019).

48. Hodgkin, A. L. J. J. P.: A qualitative description of membrane current and its application to conduction and excitation in nerve. 117 (1952).

49. Tanabe, S., Sato, S.,Pakdaman, K.: Response of an ensemble of noisy neuron models to a single input. Phys Rev E Stat Phys Plasmas Fluids Relat Interdiscip Topics 60 (6 Pt B), 7235-7238 (1999).

50. Destexhe, A., Mainen, Z. F.,Sejnowski, T. J.: Synthesis of models for excitable membranes, synaptic transmission and neuromodulation using a common kinetic formalism. J Comput Neurosci 1 (3), 195-230 (1994).

51. Hansel, D., Mato, G.,Meunier, C. J. E.: Phase Dynamics for Weakly Coupled Hodgkin-Huxley Neurons. 23 (5), 367 (2007).

52. Xu, Y., Ma, J., Zhan, X., Yang, L.,Jia, Y.: Temperature effect on memristive ion channels. Cogn Neurodyn 13 (6), 601-611 (2019).

53. Rezaei, H., Aertsen, A., Kumar, A.,Valizadeh, A.: Facilitating the propagation of spiking activity in feedforward networks by including feedback. PLoS Comput Biol 16 (8), e1008033 (2020).

54. Moldakarimov, S., Bazhenov, M.,Sejnowski, T. J.: Feedback stabilizes propagation of synchronous spiking in cortical neural networks. Proc Natl Acad Sci U S A 112 (8), 2545-2550 (2015). 